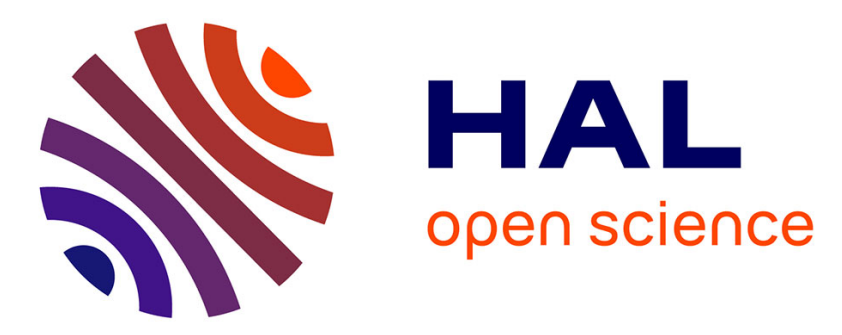

\title{
Modeling the Cholinergic Innervation in the Infant Cortico-Hippocampal System and its Contribution to Early Memory Development and Attention.
}

Alexandre Pitti, Yasuo Kuniyoshi

\section{- To cite this version:}

Alexandre Pitti, Yasuo Kuniyoshi. Modeling the Cholinergic Innervation in the Infant CorticoHippocampal System and its Contribution to Early Memory Development and Attention.. International Joint Conference on Neural Networks IJCNN 2011, 2011, San-Jose, United States. pp.14091416. hal-00750482

\author{
HAL Id: hal-00750482 \\ https://hal.science/hal-00750482
}

Submitted on 10 Nov 2012

HAL is a multi-disciplinary open access archive for the deposit and dissemination of scientific research documents, whether they are published or not. The documents may come from teaching and research institutions in France or abroad, or from public or private research centers.
L'archive ouverte pluridisciplinaire HAL, est destinée au dépôt et à la diffusion de documents scientifiques de niveau recherche, publiés ou non, émanant des établissements d'enseignement et de recherche français ou étrangers, des laboratoires publics ou privés. 


\title{
Modeling the Cholinergic Innervation in the Infant Cortico-Hippocampal System and its Contribution to Early Memory Development and Attention
}

\author{
Alexandre Pitti and Yasuo Kuniyoshi
}

\begin{abstract}
Infants present impressive developmental changes during the first year in almost all domains marked by memory categorization and variability. We propose that one important actor for this developmental shift is the cholinergic innervation of the cortico-hippocampal circuits. Based on neurological observations and developmental studies done in infants, we model how the neuromodulator acetylcholine could be gradually released from the fetal period till the first year in the hippocampal system to support the detection and the sustaining of novel signals. By doing so, the cholinergic system realizes the functional reorganization of the cortico-hippocampal system which can progressively operate then as a working memory for novelty.
\end{abstract}

\section{INTRODUCTION}

A MONG the principal neuromodulators, acetylcholine (ACh) plays a particular role on the human developing brain and thus, on the acquisition of cognitive capabilities. During early postnatal development, ACh regulates critical aspects of maturation and plasticity of the neocortex, hippocampus and cerebellum for memory and learning [2], [3], [4].

For instance, prenatal choline suppletion, which is a precursor of acetylcholine, causes long-lasting improvements in spatial memory whereas choline deficiency is associated with poor performance in certain cognitive tasks [5], [6]. Although the mechanism by which choline influences learning and memory remains unclear, converging evidences attribute a developmental role to $\mathrm{ACh}$ and suggest that it may involve changes to the hippocampal cholinergic system. In this paper, we propose that ACh operates as a kind of "order parameter" for memory development that reorganizes functionally the cortico-hippocampal system into a working memory.

The cholinergic system is composed of two chemical families with different genes expressions that have high affinity either with nicotine or muscarine via nicotinic acetylcholine receptors (nAChRs) and muscarinic acetylcholine receptors (mAChRs). Current researches in pharmacology focus their attention especially on nAChRs because of its high sensitivity with nicotine which can exert neurotoxic effects on development [7]. Prenatal and early postnatal exposures to tobacco smoke can result in altered morphological features in the

Alexandre Pitti and Yasuo Kuniyoshi are with the ERATO Synergistic Intelligence Asada Project, JST in the Department of Mechano-Informatics, Graduate School of Information Science and Technology, University of Tokyo, Tokyo, Japan (email: \{alex, kuniyosh\}@isi.imi.i.u-tokyo.ac.jp).

This work was supported by JST ERATO Asada Project developing hippocampus and cortex that can impact longterm cognitive deficits [8]. This is particularly detrimental because acetylcholine modulates brain development during critical periods when brain maturation is most sensitive to perturbation.

Innervation of nicotinic receptors $\mathrm{nAChRs}$ in the cerebral cortex and hippocampus is very early and rapid as it falls within the first six months of life [9], [1], [7]. Their roles however are found important as they regulate synaptic transmission and plasticity [10]. Besides, abnormalities in nicotinic acetylcholine receptors may relate to disruptions in cerebral circuitry development while their genetic dysfunctions have been implicated as a major feature in the neurochemical pathology of autism [7]. In contrast, the binding of muscarinic acetylcholine receptors mAChRs tends to rise significantly after birth till the first year and particularly in the entorhinal cortex - which is the gating pathway to hippocampus- to reach $80 \%$ density corresponding to the childhood period [9], [1]. Their roles differ from nAChRs but are equally important since $\mathrm{mAChRs}$ regulate the maturing entorhinal system to detect and to support the encoding of novel signals into the hippocampus [11]. Taken together, these findings suggest that the understanding of the innervation timeline of the cholinergic system into the various brain regions, more marked in the hippocampal system, can provide us a better comprehension of the developmental changes occurring during the first year.

From a cognitive viewpoint, the cholinergic system is known to regulate the balance between memory storage and renewal depending on its concentration level and the brain regions where it is released. In the cerebral cortex, ACh modulates the synaptic plasticity by enhancing long-term potentiation depending on its concentrate rate [12]. In the hippocampal system, ACh acts as a working memory for novel information [4], [11]; high concentration level of ACh sets the circuit dynamics for attention and encoding of new memory whereas low level of ACh regulates the consolidation of older memories [13]. More precisely, mAChRs are involved in the persistent firing of individual entorhinal neurons for the maintenance of novel information [14], [15], [16] and nAChRs are involved in synaptic plasticity of the hippocampal cells for learning memory patterns.

Interestingly, the period of cholinergic maturation in the hippocampal system coincides with the period when infants enrich their motor repertoire with novel actions [17], categorize novel objects into new classes [18], [19], shift from an 
Developmental Scenario for Early Memory and Attention
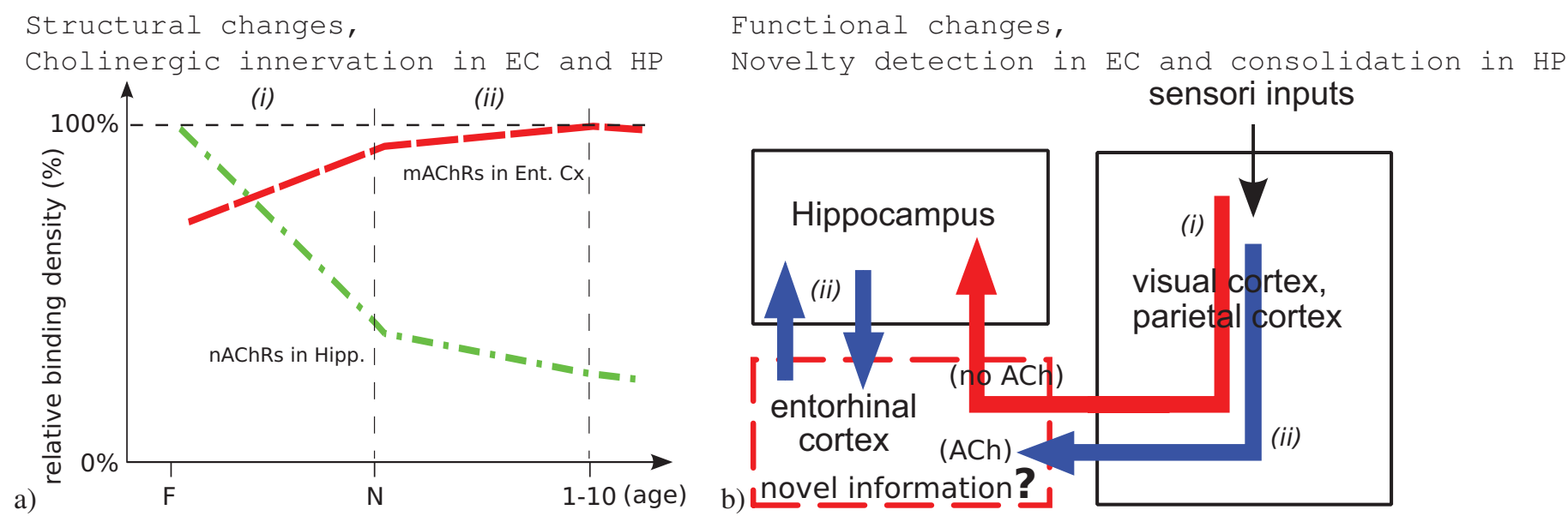

Fig. 1. Structural and functional changes in the hippocampal system and its contribution for early memory development and attention. The graph a) retranscribes the cholinergic changes in the entorhinal cortex and in the hippocampus (CA3 and CA1), adapted from [1]. Figure b) presents our hypothesis on the functional activation of the hippocampal structure after maturation of the cholinergic system during the first year, period (ii).

egocentric represention of space to an allocentric one [20] which are all features attributed to hippocampal processing. Furthermore, this chronology agrees with Nelson's proposal and others that the brain systems responsible for adult-like explicit memory, including the hippocampus and surrounding cortex, do not come online until the second half of the first year of life [21] and that infants rely on different types of learning systems during the first year [22], [17]. One might envision therefore the cholinergic system to activate rapidly the learning capabilities of the hippocampal system (i.e., fast nAChRs binding) while it regulates slowly the filtering capabilities of the entorhinal system for novelty detection (i.e., slow mAChRs binding), see Fig. 1. We think that these two parallel processes change the functionality of the hippocampus into an efficient working memory dealing with novelty, which is not at birth.

The paper is organized as follows. In the first part we define the networks architecture of the para-hippocampal system and the neuromodulatory mechanism of the cholinergic system that regulates learning and attention. The good balance between these rules controls the overall stability and plasticity of the system to maintain top-down hippocampal signals and to sustain the novel ones coming from the entorhinal system (i.e., novelty detection and support of bottom-up signals). It follows that, without $\mathrm{ACh}$, the cortico-hippocampal system behaves as a classical associative memory that extracts the statistical features from the inputs; e.g., a probabilistic network based on statistical learning. In contrast, the gradual activation of ACh changes the cortico-hippocampal system into a self-organizing map that rewards the novel signals over the familiar patterns; e.g., a hierarchical memory map such as a Bayesian tree. The new system acquires the emerging functionalities of a working memory dealing with novelty by categorizing the novel patterns and by maintaining them active during encoding. Hasselmo suggests that this feature could underlie the intrinsic mechanism for delayed-response tasks for novel stimuli in the para-hippocampal cortices even over $8-10 \mathrm{sec}$ whereas other brain regions seem to be sufficient for normal delayed matching function with small numbers of highly familiar stimuli [11].

\section{Neuromodulators and NeUral Circuits}

We describe in this section the models defined for the hippocampus and the entorhinal cortex with their respective networks architecture and regulatory mechanisms.

\section{A. Stellate Cells of Entorhinal Neurons}

In comparison with the most common types of neurons in the neo-cortex, the stellate cells of entorhinal neurons possess some specific internal currents, cholinergic-dependent, which permit them to sustain long-lasting bursting even if inputs fade away [15], [23]. Moreover, the entorhinal neurons possess very few recurrent connections so that the entorhinal cortex can be modeled as a segregated network of isolated neurons with no synaptic connections between them. According to Hasselmo, it is the muscarinic cholinergic receptors that enable persistent spiking to continue after the sample stimulus [4], [11]. We formalize them with the neuron model proposed by Izhikevich [24] which is a resonator cell whose bursting frequency increases depending on the input current $I$ :

$$
\left\{\begin{array}{l}
C v^{\prime}=k\left(v-v_{r}\right)\left(v-v_{t}\right)-u+I \\
u^{\prime}=a b\left(v-v_{r}\right)-u
\end{array}\right.
$$

where $I$ is the external input bound in the interval $[0 ; 2000 \mu A], v$ represents the membrane potential of the neuron and $u$ represents a membrane recovery variable (c.f., [24], [25]); $v^{\prime}$ and $u^{\prime}$ their temporal derivate. The voltage threshold level $v_{t}$ is set to $-45 \mathrm{mV}$ and the resetting voltage level $V_{r}$ to $-60 \mathrm{mV}$. We set also the constants $C=2000$ and $k=0.75$. The after-spike resetting is done with 


$$
\text { if } v \geq+v_{\text {peak }}, \text { then }\left\{\begin{array}{l}
v \leftarrow c \\
u \leftarrow u+d .
\end{array}\right.
$$

with $v_{\text {peak }}=30 \mathrm{mV}$. The variables set $\{a, b, c, d\}$ defines the neurons attributes $(a ; b)=(0.01 ; 15.0)$ and $(c ; d)=$ $(-50 ; 200)$.

\section{B. Hippocampal Neurons}

We define the hippocampal neurons with the model proposed by Colliaux [26] that realizes a up- and down-states where up-states are associated with firings and down-states with silences. Up- and down-states occur at each theta cycle - which is the natural rhythm of the hippocampal neurons betwen $6-10 \mathrm{~Hz}$ - and the up-state firings trigger in advance or in retard to it. The phasic (temporal) information from all the neurons represent then one memory pattern. The model consists of two coupled variables, $S$ and $\phi$, such that an oscillation component $\cos \phi$ produces the intrinsic oscillation of the membrane potential $S$ for which the phase $\phi$ depends on its level of depolarization. In a network of $N$ units, the state of the cell $i$ is defined by $\left\{S_{i}, \phi_{i}\right\} \in \Re \times[0,2 \pi[$ $(i \in[1, N])$ and evolves according to the dynamics:

$$
\left\{\begin{array}{l}
S_{i}^{\prime}=-S_{i}+\frac{1}{N} \sum_{j=1}^{N} w_{i j} R\left(S_{j}\right)+\Gamma\left(\phi_{i}\right)+I_{i} \\
\phi_{i}^{\prime}=\omega+\left(\beta-\Lambda\left(S_{i}\right)\right) \sin \phi_{i}
\end{array}\right.
$$

with $w_{i j}$, the synaptic weight between cells $\mathrm{i}$ and $\mathrm{j} . R\left(S_{j}\right)$ is the spike density of the cell $j$ and $I_{i}$ represents the driving stimulus which enables to selectively activate a cell. In the second equation, $\omega$ and $\beta$ are respectively the frequency and the stabilization coefficient of the internal oscillation.

The spike density is defined by a sigmoid function:

$$
R(x)=\frac{1}{2}(\tanh (g(x-0.5))+1) .
$$

The coupling between the two equations, $\Gamma$ and $\Lambda$ appear as follows:

$$
\left\{\begin{array}{l}
\Gamma\left(\phi_{i}\right)=\sigma\left(\cos \phi_{i}-\cos \phi_{0}\right) \\
\Lambda\left(S_{i}\right)=\rho S_{i}
\end{array}\right.
$$

where $\rho$ and $\sigma$ modulates the coupling between the internal oscillation and the membrane potential, and $\phi_{0}$ is the equilibrium phase obtained when all cells are silent $\left(S_{i}=0\right)$; i.e., $\phi_{0}=\arcsin (-\omega / \beta)$. We used the following parameters in our experiments: $\omega=1, \beta=1.2$ and $g=10$. Accordingly, $\cos \phi_{0} \approx-0.55 . \rho, \sigma$ are adjusted respectively to 1 and 0.96 , and external voltages $I$ are normalized below 0.1 to not saturate the hippocampal dynamics.

The coupling to the entorhinal system is done as follows. The entorhinal neurons receive the membrane voltage $S$ from their respective hippocampal neurons, which is originally comprised between $[-0.5 \mathrm{~V} ; 1.5 \mathrm{~V}]$ and renormalized to $[0 m A ; 2000 m A]$, such that any up-state oscillation entrains the entorhinal neuron to increase its firing rate.

The system behaves as follows. For a hippocampal network of eighty units $(N=80)$ regrouped into ten clusters with initial synaptic weights, the system transits freely from one pattern to another without external inputs, see the raster

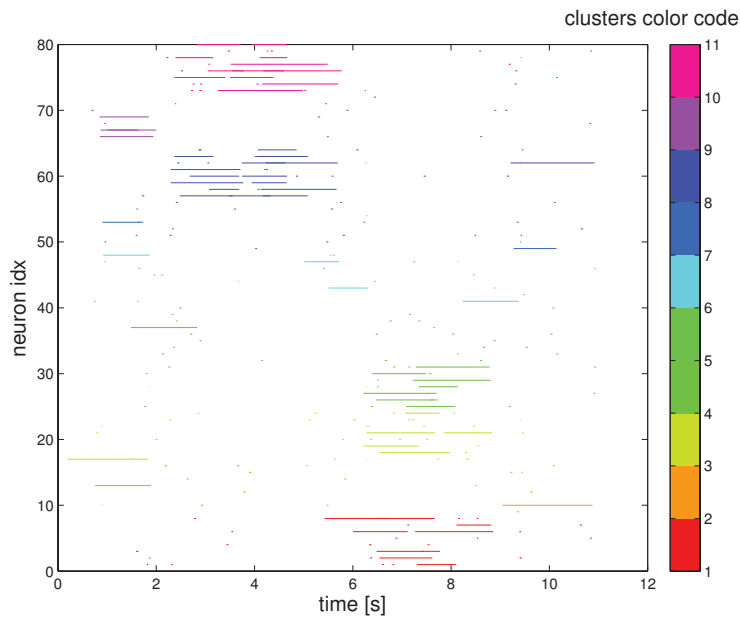

Fig. 2. Raster plot of the hippocampal dynamics without external drive. The network dynamics stabilize or switch from one cluster to another at each theta cycle.

plot in Fig. 2; same conditions as in [26]. The small perturbations pull up one pattern (up-state) and pull down the others (down state) at each theta cycle. Under these conditions, the coupled system has a poor stability-plasticity trade-off as it can recall rapidly its already learnt patterns but cannot maintain easily the new ones.

\section{Hebbian learning}

Memory patterns in the hippocampal system are associated with the respective up-state of the active cells, see Fig. 2. The robustness of one particular neural pattern depends then on the strength of the neurons' synaptic weights. The regulation of these weights are done by the classical hebbian rule that strengthens the links of two contingent neurons $i$ and $j$ by a small fraction $\Delta w$ (long-term potentiation) computed by

$$
\Delta w=\epsilon I_{i} I_{j},
$$

with learning rate $\epsilon=10^{-5}$. The weights' updating rule is then:

$$
w_{i j}(t+1)=w_{i j}(t)+\Delta w .
$$

The hippocampal system behaves as a classical associative memory which follows the probability distribution of the imposed external inputs. The exposure to random inputs - e.g., a Poisson distribution of 1 spike per millisecondwill drive slowly the hippocampal weights from their initial configurations to follow the inputs' probability distribution (not plotted here). Hence, without any attentional mechanism to sustain novel information, the network is too weak to learn novel inputs in one-shot exposure but it is robust enough to persevere to familiar dynamics.

\section{Cholinergic neuromodulation}

Acetylcholine levels control the balance between memory storage and memory update at both the cellular and circuits levels [11]. In the hippocampus, nAChRs modulate the 
synapses' plasticity at the circuit level whereas mAChRs modulate the neurons firing at the cells level in the entorhinal cortex: on the one hand, when a novel input is exposed, high cholinergic levels make the entorhinal cells to generate spikes and favor hippocampal plasticity; i.e., plasticity to afferent input, on the other hand, when a familiar input is exposed, low cholinergic levels unaffect the entorhinal cells and consolidate the hippocampal network; i.e., robustness against afferent input. This mechanism could be very important for maintaining responsiveness to sensory input in attentional tasks and for encoding new memories.

The concentration level of ACh can be defined then as the relative novelty index of one input pattern $I$ to the embedded hippocampal patterns. The novelty distance can be computed from the hippocampal weights $w$ of dimension $N^{2}\left(w_{i, j} \in[1, \ldots, N] \times[1, \ldots, N]\right)$. For an input $I$ of $N$ elements $\left(I_{i \in[1, \ldots, N]}\right)$, the novelty index ACh_level is defined as:

$$
\text { ACh_level }=1-\frac{1}{N(N-1)} \sum_{i} \sum_{j} I_{i} \cdot w_{i j}, \text { for } i \neq j .
$$

with ACh_level comprised between $[0,1]$ for which a low value corresponds to a familiar pattern and a high value corresponds to a novel one.

Its action on the networks is as follows. In the entorhinal network, input currents are sustained for any concentration rates above a specified level novelty_threshold. One simple rule to relate the entorhinal neurons' resonance to ACh_level is:

\section{if ACh_level > novelty_threshold, then sustain input current intensity $I$.}

This condition applies for the first time the input is above the threshold and its value stays fixed during the whole period when ACh_level > novelty_threshold. In the hippocampal network, the plasticity of the hippocampal weights is adjusted proportionally to ACh_level which functions as a variable learning rate [27]. The updating rule in eq. 7 is changed in:

$$
w_{i j}(t+1)=w_{i j}(t)+\text { ACh_level } \Delta w .
$$

To understand better the effect of neuromodulation on the networks, we reconduct a priming task experiment when the networks EC-HP are exposed to a novel input, see Fig. 3. The physiological mechanism responsible for the recognition of novelty and stabilization of input pattern in EC has been identified as the ACh dependent's intracellular Alonso current [11]. We simulate its effect on the HP-EC networks. We recall that HP and EC have eigthy units each with intramap connections for HP (not for EC) and that EC units have unidirectional connections to their respective HP units.

The presentation of a novel input to the entorhinal cortex at $t=186.25 \mathrm{~s}$ (see Fig. $3 \mathrm{~b}$ ) automatically rises ACh_level to a high value (see the blue crosses in Fig. 3 a). ACh's
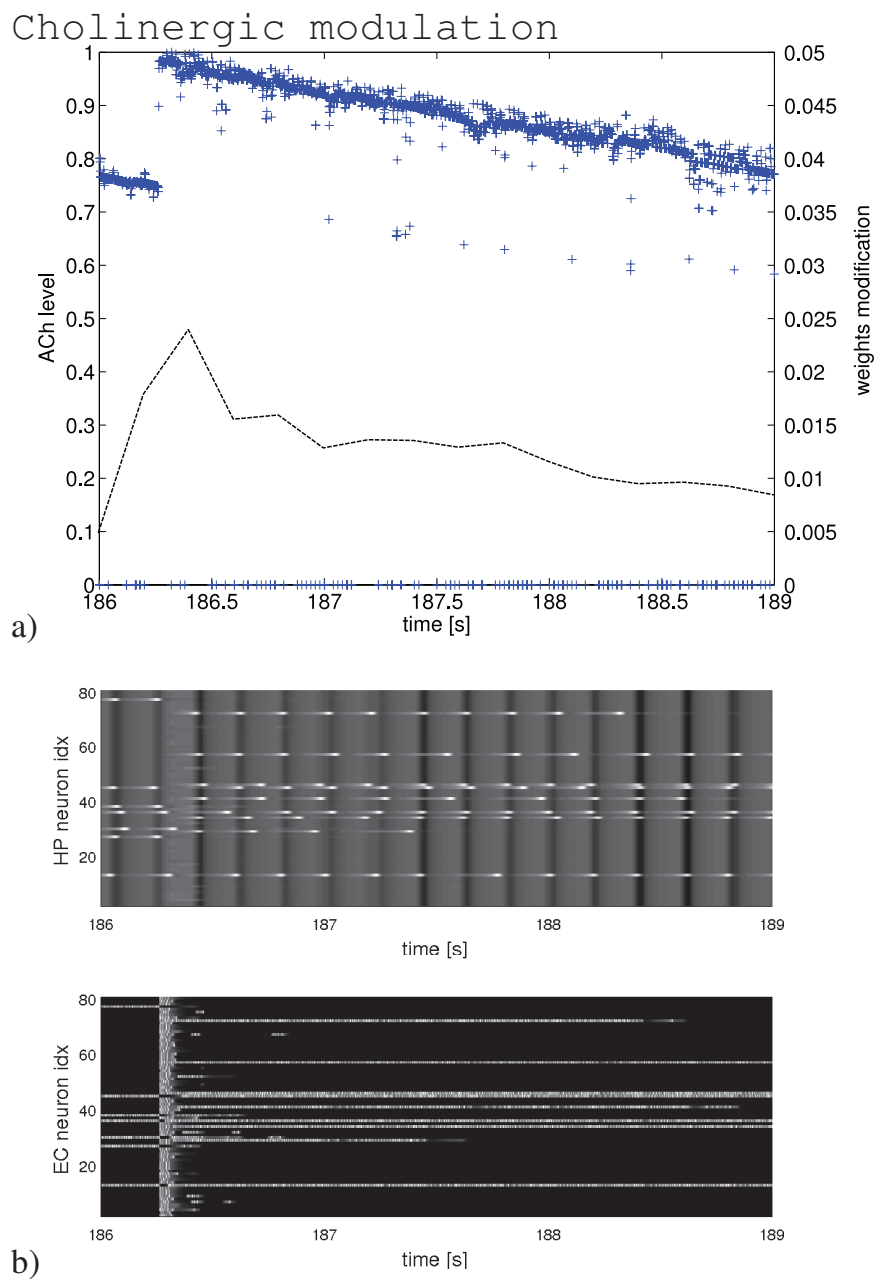

Fig. 3. Effect of cholinergic activation on the hippocampal and entorhinal dynamics after presentation of a novel input. The exposure of a novel input rises ACh level to its highest value [blue crosses in a)], which contributes to sustain the dynamics in both networks and to enhance its learning. While the pattern is being learnt, ACh level slowly decreases as a counter-effect.

increasing has for net effect to keep the entorhinal neurons firing for several seconds and to enhance the hippocampal encoding of the novel memory with respect to eq. 9, see the black line in Fig. 3 a). Therefore, the more novel a pattern is, the higher ACh_level (ascending phase). In return, the hippocampus strengthens more its synaptic links to the novel pattern, which makes it less novel and reduces accordingly $\langle\Delta w\rangle$ and ACh_level (descending phase). Thus, this process produces a temporal cost that depends on the variables ACh_level, novelty_threshold, $\Delta w$ and $I$. In other words, it plays the role of a retainer that can be used further for attentional purposes, either for learning one novel memory or assessing one old memory. The cholinergicallyenhanced network operates therefore very differently from its normal regime showed in Section II-C.

We think that the neural development of acetylcholine into the hippocampal system is related to the cognitive development in memory and categorization tasks observed during the postnatal period. 


\section{EXPERIMENTS}

\section{A. Developmental timeline of cholinergic innervation}

In the previous section, we presented how the hippocampal network behaves with and without cholinergic neuromodulation. Here we design the progressive cholinergic innervation into the hippocampal system. Muscarinic binding in the entorhinal cortex -responsible for sustaining novel signalsis slow to mature and reaches its highest level at one year-old period [1]. Besides, nicotinic binding in the hippocampus responsible for reinforcement learning- is very fast to mature as it drastically falls with age during the fetal and postnatal period [1], [8]. We model the gradual mAChRs binding in EC with the variable activation_ratio that grows continuously within the range $[0,1]$ : activ._ratio $=0$ corresponds to the fetal period when there is no muscarinic binding and activ._ratio $=1$ corresponds roughly to the period when the infant reaches its first year.

To simplify our experimental setup, the growing parameter is set to augment linearly with a step of $10^{-4}$ per iteration $(1 \mathrm{~ms})$, starting at $t=50 \mathrm{sec}$. The variables ACh_level and novelty_threshold are weighted to it so that they reach progressively their maximal value when activation_ratio $=1$. The initial level of novelty_threshold is set to 0.85 to filter as much as possible novel signals in the beginning. During the transitory period, we decrease its value to allow more novel signals to pass. This is the unique ad hoc rule that we impose to the system to simulate the functional acceleration observed during cholinergic innervation. The equations set for the cholinergic activation is then:

$$
\left\{\begin{array}{l}
\text { ACh_level }(t+1)=\text { activ_ratio } \times \text { ACh_level }(t) \\
\text { novelty_threshold }(t+1)= \\
\text { activ_ratio } \times(0.85-0.25 \text { activ_ratio })
\end{array}\right.
$$

where activ._ratio stands for activation_ratio. We present in Fig. 4 the results of this developmental scenario when the hippocampal system is exposed to random inputs, starting at $t=50 \mathrm{sec}$, when activ._ratio increases linearly to 1 .

Fig. 4 a) plots the weights modification $\langle\Delta w\rangle$ averaged over all the weights at each time step, Fig. 4 b) plots the evolution of ACh_level during cholinergic activation and Fig. 4 c) displays the overall complexity inside the hippocampal network computed from the synaptic weight matrix. The complexity measure $C(w)$ of the system's weight matrix $w$ is defined as the difference between the integration level $I(w)$ considered as a whole and the average integration for small subsets within, following [28]: $C_{N}(w)=$ $\sum_{k=1}^{n}\left[(k / n) I(w)-\left\langle I\left(w_{j}^{k}\right)\right\rangle\right]$. Low complexity levels reflect a poorly organized network with low memory capacity (a uniform distribution) whereas high levels reflect functional connectivity within the network and higher memory capacity.

The situation before ACh activation when activ._ratio $=0(t<50 \mathrm{sec})$ corresponds to the
Cholinergic activation

activation_ratio

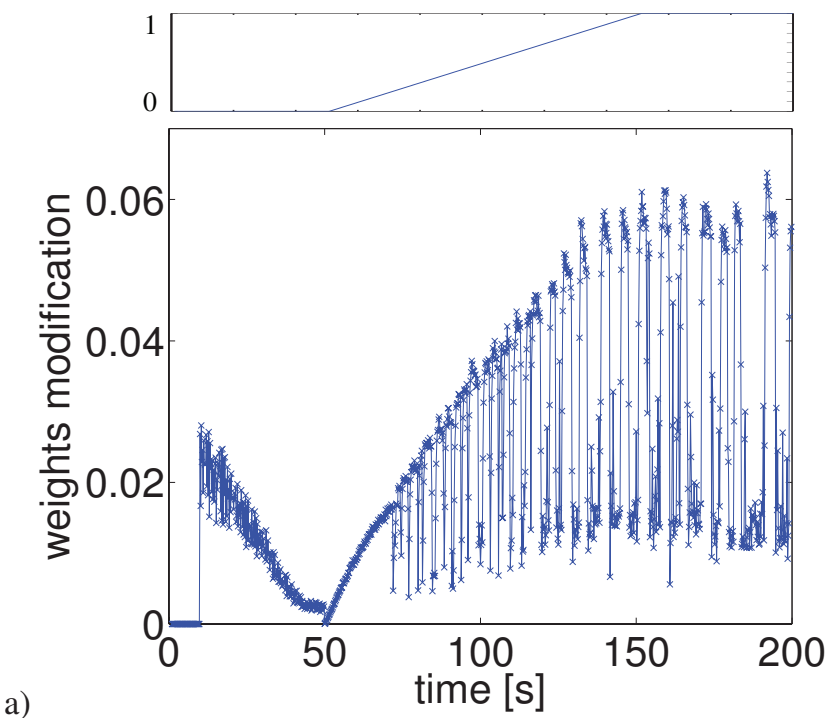

b)
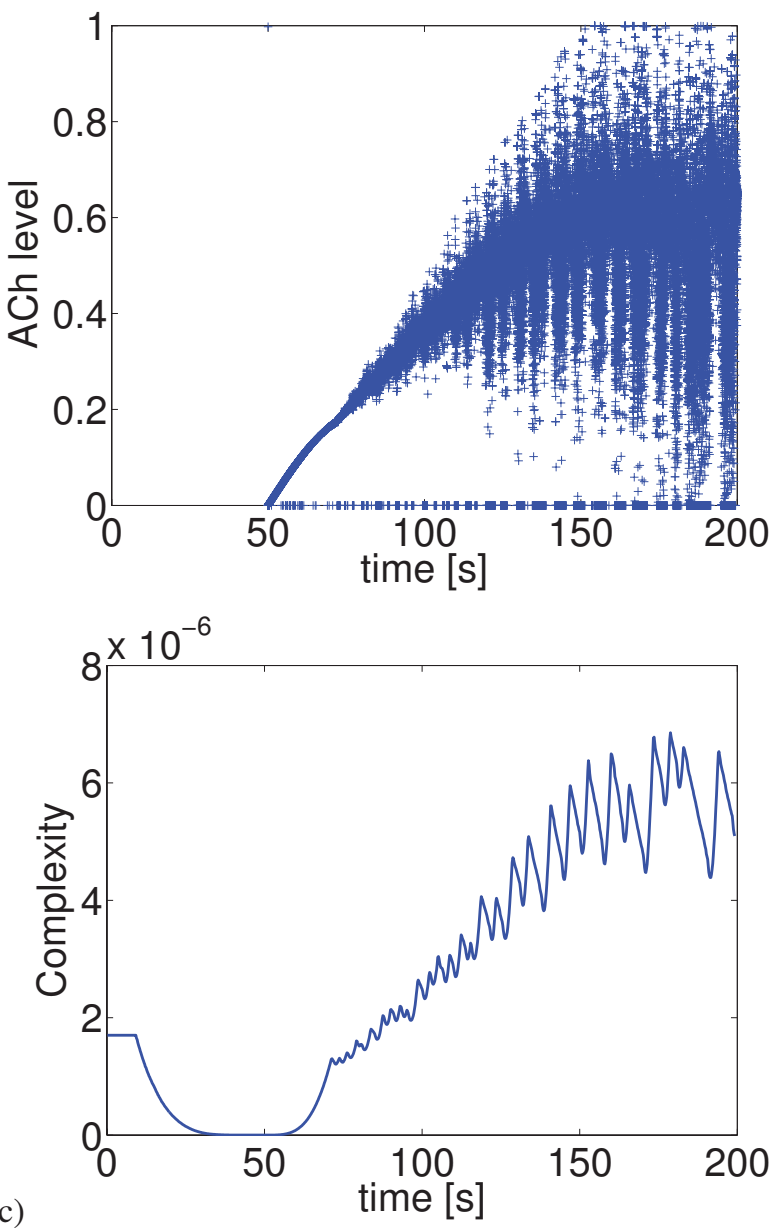

Fig. 4. Weight modication for Hebbian learning with gradual activation of ACh modulation starting at $t=50 \mathrm{sec}$ resp. a) and b). Complexity measures computed from the connection matrix of the hippocampal network relative to cholinergic activation rate (c). 
case described in Section II-C when the learning system encodes continuously the external inputs and converges to its probabilistic distribution. The weights rapidly stabilize themselves within a minimal fluctuation regime and the system behaves as a classical associative memory. Parallely, the complexity measure decreases continuously indicating the hippocampal structure always adapt itself to the novel inputs but do not retain them for a long period.

At contrary, the activation of the cholinergic variables when activ._ratio $>0$ at $t=50 \mathrm{sec}$ reverses the process and generates a phase transition within the neural dynamics. Here, the variable ACh_level starts to oscillate between low and high states with bigger amplitudes as activ._ratio augments: the learning system becomes more and more sensitive to the inputs' novelty and scaffolds its memory capacity by embedding one at a time a novel input. The ascending phases (i.e., the crests) correspond to the encoding periods and the descending phases (i.e., the troughs) correspond to the consolidation periods.

Moreover, the capability to detect and to encode novel inputs creates some new emergent functionalities within the system. For instance, since the new patterns do not overlap with the old memories, the hippocampal system tends to be sparsely organized, which rise in fine its complexity level. In line with Hasselmo's proposal [4], this mechanism could prevent interference from previously stored patterns during the learning of new patterns. In other words, it enables the hippocampal system to categorize on the fly new memories while preserving the old ones. The result is the emergence of a 'working memory' that scaffolds over time, the memory organization is kept motivated for novelty and reaches a high complexity level.

When the learning system reaches its fullest capacity (i.e., its highest complexity level), it attains the so-called plasticity/stability limit where it cannot embed new memories without erasing old ones. Despite this unavoidable trade-off, ACh mechanism slows down memory decays in comparison to the situation without, see Fig. 5. From a system theory viewpoint, $\mathrm{ACh}$ acts therefore as a kind of low-pass filter that retains information.

Moreover, ACh plays a similar function during memory encoding as it slows down the scattering of one novel pattern by sustaining its trace in EC; the memory retention of one novel signal depends then indirectly on activ._ratio, see Fig. 6. The time duration for holding one novel pattern augments with activation_ratio to reach 6 or 7 seconds when ACh is fully active. As a result, these two mechanisms permit to create a working memory (i.e., an attentional system) that can serve advantegeously when the system is dealing with delays or with unexpected events; e.g., to sustain object continuity during objects' rotation and occlusion.

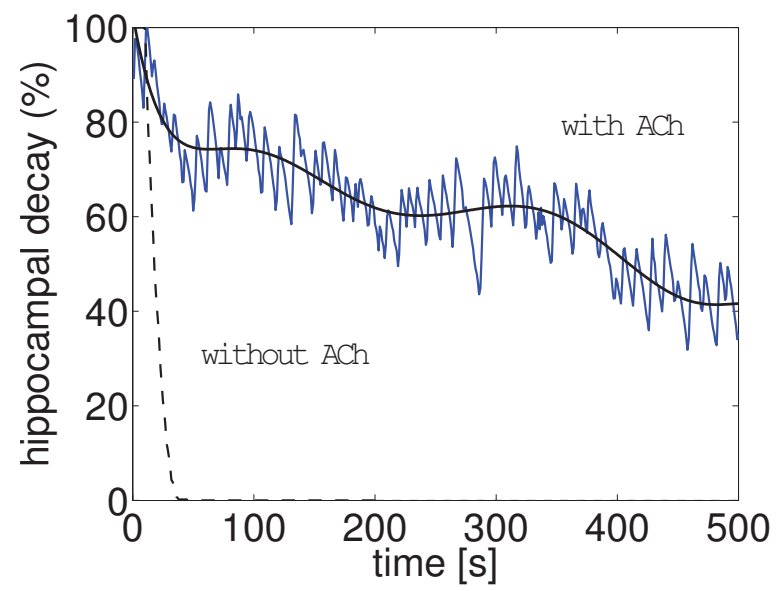

Fig. 5. Hippocampal memory retention and decay. Without cholinergic activation, the newly acquired memories in the hippocampus vanish asymptotically and are replaced by other memories whereas the ACh mechanism augments the retention time with a slow decay.

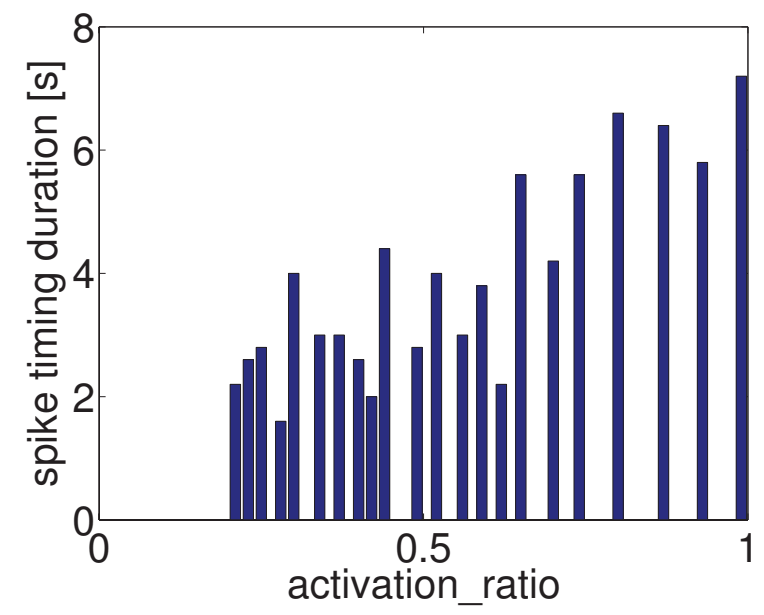

Fig. 6. Time duration relative to activation_ratio. It shows the performance of the working memory in terms of time duration to hold novel information. The spike duration correlates almost linearly with the values of the activation parameter.

\section{B. Memory retention, transfer and consolidation into the neo-cortex}

ACh creates the conditions for the rapid formation of memory traces in the hippocampus and allows the retention of specific episodes while preventing as much as possible interferences. However, in order to avoid memory decay, the hippocampus has to reinstantiate the short-term memories into the neo-cortical maps, which are performing at a slower learning rate [22]. When the later structures finish to consolidate the memory patterns, they can start manipulate them ad libitum without the need of the hippocampus.

The role of acetyholine is however not impotent in this scheme. For instance, Peinado demonstrated that ACh mediates the propagation of slow waves of electrical activity in the developing neocortex [29]. We propose to model the memory remapping from short-term hippocampal memories to long-term cortical memories.

Cortical maps are commonly defined as networks with 
recurrent connections. We use as neuron model a variant of the stellate cell defined in eq. 2, also proposed by [24]:

$$
\left\{\begin{array}{l}
v^{\prime}=0.04 v^{2}+5 v+140-u+I \\
u^{\prime}=a(b v-u)
\end{array}\right.
$$

where $I$ is the external input. The auxiliary after-spike resetting equation is the same as eq. 2 with $v_{\text {peak }}=$ $30 \mathrm{mV}$ such as the variables $v$ and $u$ represent also the membrane potential of the neuron and the membrane recovery variable. The variables set $\{a, b, c, d\}$ defines the neurons attributes whether excitatory $(a ; b)=(0.02 ; 0.2)$ and $(c ; d)=(-65 ; 8)$, or inhibitory; $(a ; b)=(0.02 ; 0.25)$ and $(c ; d)=(-65 ; 2)$.

The hebbian learning is based on the mechanism of spike timing-dependent plasticity introduced in [25] and each neuron receives the external current $I$ from their respective entorhinal neurons $I_{\text {ent }}\left(I_{\text {ent }}=20 \mathrm{~mA}\right.$ when the neuron fires) and pre-synaptic neurons $i: I=\sum_{i \in \text { pre }} I_{i} . w_{i}+I_{\text {ent }}$. The cortical map is composed of 800 excitatory neurons and 200 inhibitory neurons with a coupling probability distribution of 0.1 (10 connections per neuron) for all the neurons. Therefore, the cortical map is ten time bigger than the entorhinal system - which has only eighty neurons- and each entorhinal cell is exactly connected to only one excitatory cortical neuron with index corresponding to the entorhinal neuron index multiplied by 10 . The other cortical neurons are therefore inter-neurons that receive indirect signals from their recurrent connections.

During memory consolidation, we evaluate the rate of cortical encoding with the Victor-Purpura metric distance (VP) that quantifies the similarity between two spike trains [30]; e.g., the signal/noise ratio of one pattern. We use this measure because probabilistic methods are more approximative as they require to average the signals into spike rates. The VP distance computes the minimal cost $D_{v}$ of transforming $x$ to $x^{\prime}$ using two basic operations: the event insertion or deletion with a cost equals 1 and the event movement for which the cost is proportional to the distance (constance $C_{V}$ ); the time constant is defined as $\tau_{V}=1 / C_{V}$; here $C_{V}=1$. In the case that the two spike trains are identical, then we will have $D_{V}=0$.

We define the memory retention inside the cortical map as the inverse of $D_{V}$ renormalized between 0 to $100 \%$, calculated from the hippocampal and the cortical dynamics. Under this condition, the more the cortico-hippocampal spike trains are synchronous, the more the cortical map is accurate and the higher is the memory retention.

We present in Fig. 7 the retention score of the cortical map when exposed to hippocampal dynamics and a raster plot of cortico-hippocampal synchronization during memory consolidation/retrieval in Fig. 8. In comparison with the performance of the hippocampus for learning one memory in one-shot and very rapidly [see Fig. 3 a)], the cortical map is much slower for encoding one memory pattern as it takes one hundred seconds to converge ( $10^{5}$ iterations). Moreover, the cortical map performs worse than HP as it reaches $70 \sim 75 \%$ retention score whereas the hippocampus can learn a very

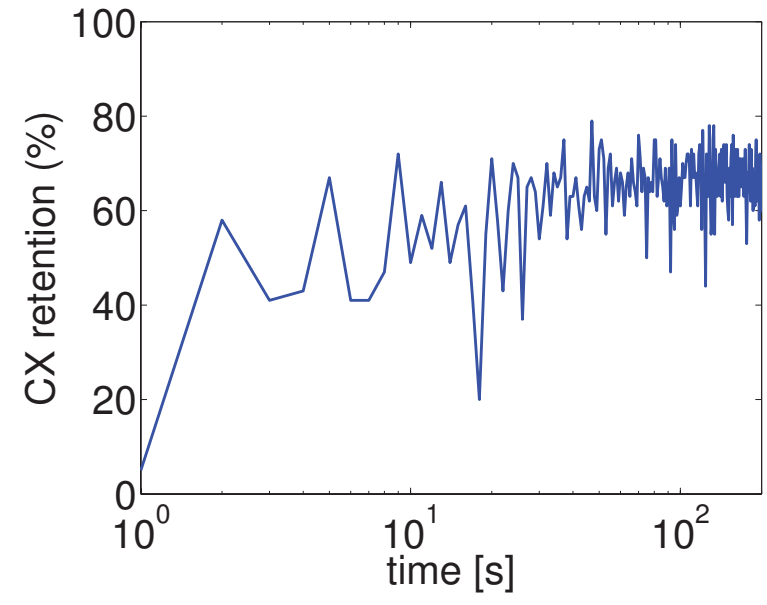

Fig. 7. Memory consolidation in the cortical map. Memory retention is estimated from the signal per noise ratio between the hippocampal items and the cortical map activity with the Victor and Purpura distance between two spike trains [30]. The cortical map learns the hippocampal patterns at a very slow rate compared with the hippocampus.
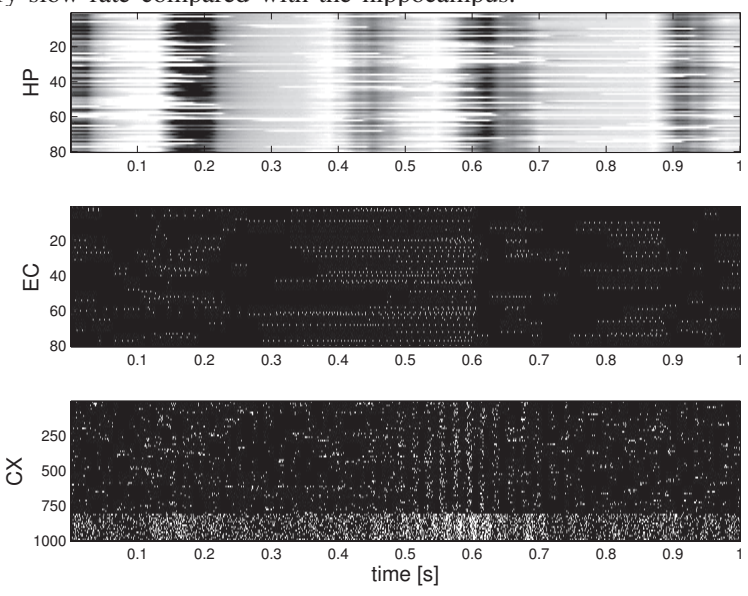

Fig. 8. Cortico-hippocampal synchronization. The raster plots of the cortico-hippocampal maps present the $\theta / \gamma$-bands synchronization of the cortical neurons to the hippocampal $\theta$ rhythm for a familiar pattern, the gamma waves enhance selectivity to the input patterns.

precise pattern without interference. The reason for this behavior comes from the recurrent connections that amplify the neural activity inside the cortical map, which performs then as an associative memory. Moreover, memory consolidation generates the theta/gamma rhythm synchronization: the rhythmic activity corresponds here to the formation of coherent clusters that can be used for memory retrieval or for other tasks such as memory association and/or anticipation.

The temporal durations of hippocampal decay (Fig. 5) and cortical encoding (Fig. 7) are therefore critical as they delineate the interval length during which a memory pattern in the hippocampus is available for cortical encoding, before being lost permanently. Like for the hippocampus, the cortical map has a trade-off in terms of plasticity and stability for consolidating the short-term memories into longterm memories that depends on the learning rates of both systems. 


\section{DISCUSSION}

In this paper, we proposed to model the cholinergic system innervation in the hippocampal system and its influence for learning, attention and memory development. Acetylcholine is involved in the structural and functional adjustments of the hippocampus, transforming it into an attentional system; i.e., a working memory for novel information. Under its action, the entorhinal cortex sustains and facilitates the learning of novel stimuli relative to the old patterns already present in the hippocampus. We show in our experiments how this dual mechanism may generate simply some emergent properties necessary for cognitive development. For instance, it limits the interference between memories which has for effect to scaffold the memory organization and to discretize the memory space into separated categories in the same time.

Our cholinergic hypothesis may give some partial answers to the paradoxes that pose the hippocampus and other subcortical structures that appear to function at birth but show some evidence of slow development and/or functional reorganization. Here, we propose that the neurotransmitter acetylcholine may play the role of a "catalyst" that activates the functional organization of the cortico-hippocampal system (i.e., detecting and holding stimuli, preserving and acquiring memories).

Although ACh is generally known to regulate the structural maturation of the central nervous system [2]- e.g., the growth, differentiation, and plasticity of the neurons- the precise timing of cholinergic innervation to the cortex appears to be crucial also for the normal development of cognitive functions. Its action is even broader since $\mathrm{ACh}$ has been identified for mediating the propagation of slow waves of electrical activity in the developing neocortex [3], [29], which are associated with long-term memory and categorization performances [18], [19]. In our model, we show how theta waves could slowly shape the neocortical maps into coherent patterns (rhythmical theta/gamma activity). Hence, the modeling of the cholinergic system in the para-hippocampal system is not only critical for understanding development during the first year [1] but also for understanding memory transfer, attention processes and retrieval task [4].

\section{REFERENCES}

[1] J. Court, S. Lloyd, M. Johnson, M. Griffiths, N. Birdsall, M. Piggott, A. Oakley, P. Ince, E. Perry, and R. Perry, "Nicotinic and muscarinic cholinergic receptor binding in the human hippocampal formation during development and aging," Developmental Brain Research, vol. 101, pp. 93-105, 1997.

[2] J. Lauder and U. Schambra, "Morphogenetic roles of acetylcholine," Environmental Health Perspectives, vol. 107, pp. 65-69, 1999.

[3] L. Descarries, N. Mechawar, N. Aznavour, and K. Watkins, "Structural determinants of the roles of acetylcholine in cerebral cortex," Progress in Brain Research, vol. 145, pp. 45-58, 2004.

[4] M. Hasselmo, "The role of acetylcholine in learning and memory," Current Opinion in Neurobiology, vol. 16, pp. 710-715, 2006.

[5] M. Matsukawa, M. Ogawa, T. Nakadate, K. Maeshima, Y. Ichitani, N. Kawai, and N. Okado, "Serotonin and acetylcholine are crucial to maintain hippocampal synapses and memory acquisition in rats," Neuroscience Letters, vol. 230, pp. 13-16, 1997.
[6] W. Meck, C. Williams, J. Cermak, and J. Blusztajn, "Developmental periods of choline sensitivity provide an ontogenetic mechanism for regulating memory capacity and age-related dementia," Front. Integr. Neurosci., vol. 1, no. 7, pp. 1-11, 2008.

[7] J. Dwyer, S. McQuown, and F. Leslie, "The dynamic effects of nicotine on the developing brain," Pharmacology \& Therapeutics, vol. 122, pp. 125-139, 2009.

[8] J. Court, C. Martin-Ruiz, A. Graham, and E. Perry, "Nicotinic receptors in human brain: topography and pathology," Journal of Chemical Neuroanatomy, vol. 20, pp. 281-298, 2000.

[9] J. Court, E. Perry, M. Johnson, M. Piggott, J. Kerwin, R. Perry, and P. Ince, "Regional patterns of cholinergic and glutamate activity in the developing and aging human brain," Developmental Brain Research, vol. 74, pp. 73-82, 1993.

[10] B. McKay, A. Placzek, and J. Dani, "Regulation of synaptic transmission and plasticity by neuronal nicotinic acetylcholine receptors," Biochemical Pharmacology, vol. 74, pp. 1120-1133, 2007.

[11] M. Hasselmo and C. Stern, "Mechanisms underlying working memory for novel information," Trends in Cognitive Sciences, vol. 10, no. 11, pp. 487-493, 2006

[12] K. Doya, "Metalearning and neuromodulation," Neural Networks, vol. 15 , p. 495506,2002

[13] M. Hasselmo and J. McGaughy, "High acetylcholine levels set circuit dynamics for attention and encoding and low acetylcholine levels set dynamics for consolidation," Progress in Brain Research, vol. 145, pp. 207-231, 2004.

[14] R. Knight, "Contribution of human hippocampal region to novelty detection," Nature, vol. 383, pp. 256-259, 1996.

[15] R. Klink and A. Alonso, "Ionic mechanisms of muscarinic depolarization in entorhinal cortex layer ii neurons," J. Neurophysiol., vol. 77, pp. 1829-1843, 1997.

[16] D. Kumaran and E. Maguire, "Novelty signals: a window into hippocampal information processing," Trends in Cognitive Sciences, vol. 13, no. 2, pp. 47-54, 2009.

[17] K. Adolph and A. Joh, Multiple learning mechanisms in the development of action. New York: Oxford University Press, 2009.

[18] L. Smith and L. Samuelson, "Perceiving and remembering: Category stability, variability and development," In K. Lamberts \& D. Shanks (Eds.), Knowledge, Concepts and Categories. Psychology Press, East Sussex, UK, pp. 161-195, 1997.

[19] P. Quinn, A. Westerlund, and C. Nelson, "Neural markers of categorization in 6-month-old infants," Psychological Science, vol. 17, pp. 59-66, 2006.

[20] N. Newcombe and J. Huttenlocher, "Development of spatial cognition," Handbook of Child Psychology, vol. 5, no. 2, pp. 734-776, 2006.

[21] C. Nelson, "The ontogeny of human memory: A cognitive neuroscience perspective," Developmental Psychology, vol. 31, pp. 723-738, 1995.

[22] J. McClelland, B. McNaughton, and R. O'Reilly, "Why there are complementary learning systems in the hippocampus and neocortex: Insights from the successes and failures of connectionist models of learning and memory," Psychological Review, vol. 102, no. 3, pp. 419457, 1995.

[23] R. Klink and A. Alonso, "Muscarinic modulation of the oscillatory and repetitive firing properties of entorhinal cortex layer ii neurons," J. Neurophysiol., vol. 77, pp. 1813-1828, 1997.

[24] E. M. Izhikevich, "Simple model of spiking neurons," IEEE Transactions on Neural Networks, vol. 14, pp. 1569- 1572, 2003.

[25] E. Izhikevich, "Polychronization: Computation with spikes," Neural Computation, vol. 18, pp. 245-282, 2006.

[26] D. Colliaux, C. Molter, and Y. Yamaguchi, "Working memory dynamics and spontaneous activity in a flip-flop oscillations network model with a milnor attractor," Cogn. Neurodyn., vol. 3, pp. 141-151, 2009.

[27] M. Hasselmo, "Neuromodulation and cortical function: modeling the physiological basis of behavior," Current Opinion in Neurobiology, vol. 67 , pp. 1-27, 1995.

[28] G. Tononi, O. Sporns, and G. Edelman, "A measure for brain complexity: Relating functional segregation and integration in the nervous system," Proc. Natl. Acad. Sci., vol. 91, pp. 5033-5037, 1994.

[29] A. Peinado, "Traveling slow waves of neural activity: a novel form of network activity in developing neocortex," J. Neurosci., vol. 20, no. RC54, pp. 1-6, 2000.

[30] J. Victor and K. Purpura, "Metric-space analysis of spike trains: theory, algorithms and application," Network, vol. 8, pp. 127-164, 1997. 